\title{
ARTE, PATRIMONIO Y ERUDICIÓN: VIRGILIO MATTONI Y LA CATEDRAL DE SEVILLA
}

\section{ERUDITION, PATRIMONIO AND THE PAINT: VIRGILIO MATTONI AND THE CATHEDRAL OF SEVILLE}

\author{
Gerardo Pérez Calero \\ Academia Iberoamericana de La Rábida. España \\ ORCID: 0000-0001-7554-5070 \\ gcalero@us.es
}

\begin{abstract}
En este trabajo se da a conocer la relación artística, patrimonial y erudita del pintor sevillano con la catedral hispalense. Para ello, se analiza su intervención como experto en pintura y restauración que, siendo académico encargado de su protección, lleva a cabo en algunas de sus más importantes dependencias. También se analiza su labor creativa en obras relacionadas exclusivamente con la seo.

Palabras clave: pintura; Sevilla; siglos XIX y XX.
\end{abstract}

In this work the artistic, patrimonial and scholarly relationship of the Sevillian painter with the hispanic cathedral is made known. For this, his intervention as an expert in painting and restoration is analyzed, which, as an academic in charge of its protection, he carries out in some of its most important dependencies. His creative work is also analyzed in works related exclusively to cathedral.

Keywords: painting; Seville; 19th and 20th centuries.

Hay que reconocer, de entrada, la identificación afectiva de un artista con su lugar de nacimiento, o con aquel que considera como el suyo propio. No son una excepción sino todo lo contrario, los sevillanos, al menos los de antaño, quienes sienten una sensibilidad especial, a veces extrema, por la singularidad de su ciudad. Virgilio Mattoni de la Fuente (1842-1923) es uno de esos artistas eruditos cuyo amor por Sevilla es manifiesto a lo largo de su vida y patente en su obra' ${ }^{1}$.

\footnotetext{
${ }^{1}$ Pérez Calero, 1977; 1996; 2005: 187-189; 2015: 387.
} 
Como se ha indicado en un trabajo reciente ${ }^{2}$, a lo largo de su dilatada biografía se desvive en alabanzas a su ciudad natal, lo que plasma en sus cuadros pintados; también por escrito, en libros, revistas, diarios, e incluso en algunas obras poéticas, pletóricos todos de desbordante emoción hacia ella. Tal vez un pensamiento suyo resuma con precisión este sentimiento: "Sevilla es el gran lienzo donde la naturaleza parece que se complace en reproducir todos sus encantos y todas sus bellezas. Su cielo diáfano, su tierra siempre florida y su clima ardiente son como la expresión del alma de sus artistas. ¡Luz, color, perfume, vida!... Y porque Sevilla es así, sus pintores también así son: luminosos, cálidos y exuberantes de sentimiento..."

En cierta ocasión manifestó públicamente que "Sevilla siempre católica fue por lo tanto siempre artística. No pudo ser por menos. La religión fue foco ardiente y luminoso que encendió e ilustró el alma y la mente de sus esclarecidos hijos. En Sevilla todos son artistas; porque artistas son todos los que saben sentir y amar y vive Dios que si de sentimientos y amores se tratase bien pudiera dar Sevilla cátedra de tal enseñanza a todos los pueblos de la tierra. Así es que nuestro arte religioso reviste un carácter especial que lo distingue del de los demás países siendo la expresiva expansión la nota dominante de esta diferencia. No podía ser de otro modo; un cielo siempre azul, un sol siempre ardiente y una tierra siempre cubierta de flores, dan por resultado el arte y la poesía"4.

Con la ceremonia de su bautismo, Mattoni inicia su relación afectiva y artística con el templo metropolitano al recibir las aguas en la pila de la iglesia parroquial del Sagrario de la Santa y Metropolitana Catedral hispalense el 30 de enero de $1842^{5}$.

Este vínculo con la Magna Hispalensis tiene para él una doble dimensión: como monumento arquitectónico, también museo catedralicio que hay que conocer y proteger, y como fuente de inspiración literaria y artística. En ambos casos, manifiesta su admiración por el que llama "arte cristiano" con el que identifica a la catedral. Dice al respecto: “¿Dónde señores existe más cumplidamente la satisfacción justa de las aspiraciones del espíritu humano, de ese bello ideal del alma que se esconde al través de lo infinito, invisible o impalpable como el germen de la vida en la esencia del ser creado, fuerza centrífuga que mueve fuerte al par que dulcemente la voluntad hacia el bien y que hace amar lo bello, lo bueno y lo verdadero, esa trinidad de cualidades que en suma no forma más que una sola,

2 Pérez Calero, 2020: 617.

${ }^{3}$ Mattoni, 1920: 127.

${ }^{4}$ Mattoni, 1890.

5 Archivo Parroquial del Sagrario de la Santa Iglesia Catedral de Sevilla (APSCSe), Libro 79, f. 242. 
puesto que en cada una de ellas se suponen incluidas todas las otras? Solamente en el arte cristiano" 6 .

Estas reflexiones no se contradicen con lo que también manifiesta en tal ocasión respecto a cualquier otro arte que no sea religioso, pues dice: "El arte por sí mismo sea en cualquiera de sus múltiples manifestaciones, siempre que cumpla con las eternas e ineludibles leyes de la dignidad, de la moralidad y de la belleza, será noble, civilizador y por tanto útil a las sociedades"7.

Veamos en primer lugar su dilatada y rigurosa intervención individual o colegiada en la catedral y después el monumento como fuente de inspiración de su obra pictórica y erudita.

\section{MATTONI: SU FORMACIÓN E INTERÉS POR EL PATRIMONIO ARTÍSTICO}

Desde muy joven, dada su vasta cultura, espíritu refinado y acendrada religiosidad, se interesó por conocer en profundidad la esencia artística de la ciudad que tanto amaba y de la que apenas se ausentó, como no fuera para realizar un viaje formativo a Roma de tres años (1872-1874). A su regreso, se detuvo en Galicia, estancia documentada por sus dibujos de algunos monumentos medievales de Santiago de Compostela y Pontevedra, que mucho le interesaron y que decantarían su gusto estético.

Es manifiesto su probado interés por el patrimonio artístico sevillano en general y su defensa desde las instituciones a las que perteneció, pero sobre todo por los dos grandes monumentos medievales: la catedral y el Real Alcázar ${ }^{8}$. Ya lo advirtió su amigo Luis Montoto al decir: "Mattoni siente dos grandes amores: el de nuestro Alcázar y el de nuestra grandiosa Catedral; y... como la dolencia de amor no bien se cura sino con la presencia y la figura, le verán Vds. a todas horas del día, embebido, absorto, contemplando las filigranas del palacio de don Pedro y las maravillas del templo metropolitano"

Sus estudios artísticos e interés por la erudición los inicia siendo estudiante en la Escuela Provincial de Bellas Artes donde tuvo como preceptor a Joaquín Domínguez Bécquer. Tampoco le fue ajena antes al contrario una profunda vinculación con Eduardo Cano, con el tiempo convertido en su maestro. Se iniciaba así su inclinación por el pasado medieval al que llegaría a admirar, estudiar e interpretar de manera concienzuda.

En 1868 se produjo la que podemos considerar su primera intervención como protector del patrimonio artístico. Ocurrió siendo un joven estudiante y por temor a una posible iconoclasia revolucionaria antirreligiosa. A este respecto,

\footnotetext{
${ }^{6}$ Mattoni, 1890.

7 Mattoni, 1890.

${ }^{8}$ Pérez Calero, 1976: 181-198.

${ }^{9}$ Montoto, 1929: 370.
} 
realizó entonces siete copias al óleo calcadas de las figuras originales del zócalo de los claustros bajos del patio de los Evangelistas del monasterio jerónimo de San Isidoro del Campo. De esta suerte -según sus propias palabras puestas en tercera persona- "Renovó en Sevilla, con algunas de sus obras, el estilo arcaico de los primitivos y quincentistas" 10 .

A su aprendizaje juvenil, Mattoni seguiría ya en su madurez la relación con estudiosos y eruditos, mayores o menores que él, algunos compañeros de instituciones y expertos archiveros. Ellos le inculcaron el amor por la Arqueología y la Historia del Arte medievales, que tanto le servirán de inspiración en sus pinturas de historia, algunas de ellas ambientadas en la propia catedral, sus alrededores, o el Real Alcázar ${ }^{11}$.

Nuestro pintor tuvo un trato muy estrecho con aquel a quien el erudito Francisco María Tubino (1833-1888) orientó en el terreno de la arqueología medieval: el polígrafo local José Gestoso y Pérez (1852-1917), diez años más joven, con quien su paisano comparte afinidades intelectuales y estéticas pese a disentir a veces con él. A este respecto, llegó a decir en una ocasión: ... por la "propensión que tiene [Mattoni] a las clasificaciones, tan expuestas a errores". Ambos conllevaban afanes y fatigas en la conservación del patrimonio artístico local, especialmente la catedral de Sevilla ${ }^{12}$.

Mattoni en Roma se imbuyó del arte y la cultura de la ciudad, lo que le inspiraría no pocos cuadros de carácter histórico recreando ambientes propios de la Antigüedad y del Medievo. Entre los primeros sobresale el titulado Las termas de Caracalla; a los segundos nos referimos en relación con la catedral como objetivo de este trabajo.

Unos años después de regresar de la ciudad eterna, realizó -como queda dicho más arriba- un viaje por Galicia del que apenas tenemos noticias si no fuera por los apuntes artísticos que ejecutó en 1879. Se trata de dibujos a lápiz de piezas de diversos enclaves monumentales: estatuas del compostelano Pórtico de la Gloria, del convento de San Francisco y de la fachada de la iglesia de Santa María, ambos de Pontevedra. De todos ellos se infiere su afición por el arte medieval.

Asentado en Sevilla y domiciliado en el entorno de la catedral y del Alcázar, comienza un cursus honorum de brillante trayectoria. Había obtenido ya medallas en varias exposiciones regionales y nacionales y desde 1886 era académico correspondiente de la de San Fernando de Madrid, así como miembro de la Comisión Provincial de Monumentos. Al año siguiente, es elegido académico de número de la de Santa Isabel de Hungría de Sevilla en sustitución del pintor y profesor José Jiménez Aranda. Estos nombramientos le responsabilizan de la integridad del patrimonio monumental, teniendo a la catedral como el ejemplar

\footnotetext{
${ }_{10}$ Mattoni, 1920: 154.

${ }^{11}$ Pérez Calero, 2020.

12 AAVV., 2020.
} 
más significativo al que dedicará toda su atención, no pocos esfuerzos y sinsabores aunque también muchas satisfacciones.

\section{EL PINTOR Y LA SEO HISPALENSE}

Desde hacía algún tiempo, el templo metropolitano necesitaba una permanente atención de vigilancia para la conservación de su monumental fábrica arquitectónica, pero también del riquísimo patrimonio artístico, verdadero museo de pintura, escultura y arte suntuaria, contenido entre sus muros y repartido por capillas y dependencias. La acción protectora estaba a cargo de la Junta de Obras de la Catedral Metropolitana, institución creada por R.O de 14 de diciembre de $1881^{13}$. De ella formaba parte un grupo de destacados especialistas entre los que se contaban los arquitectos Joaquín de la Concha Alcalde, González Rojas y el propio Virgilio Mattoni. Este último sustituyó como secretario al también arquitecto Manuel Portillo ${ }^{14}$, quien en 1886 fue a su vez sustituido por José Gestoso hasta 1896. Después lo fue Antonio Alarcón hasta el 3 de junio de 1897 en que ya lo ostenta Mattoni hasta el 3 de enero de 1919 bajo la presidencia de Marcelo Spínola y Maestre, recién elegido arzobispo de Sevilla. Fueron también miembros de la Junta: Francisco Bermúdez de Castro como presidente, y Antonio Alarcón, Joaquín Fernández y Claudio Boutelou como vocales.

Tres aciagos acontecimientos y otros de menor calado, en los que tuvo protagonismo nuestro personaje, se suceden en el templo metropolitano como referencias principales de la acción proteccionista a lo largo de otras tantas décadas: En 1888 se produjo el hundimiento del cimborrio; al año siguiente el incendio de la Capilla de la Virgen de la Antigua, y en 1909 los estragos del terremoto del mes de abril que afectaron a la iglesia parroquial del Sagrario de la Catedral.

La primera ocasión antes citada aconteció en 1888, el año de la Exposición Universal de Barcelona y el de la primera grabación de Edison. Fue con motivo del hundimiento de segmentos de algunas bóvedas cuyos restos dañaron parte del tesoro artístico cobijado en el templo. Para ello, Mattoni fue llamado por el cardenal Fray Ceferino González para hacerse cargo sobre todo del traslado para su conservación de los cuadros que estaban diseminados por los muros, reparar los que hubiese necesidad e instalarlos en espaciosos salones bajos dispuestos para tal fin en el segundo patio.

Terminaba el siglo y en el mes de abril de 1897, en su calidad de experto en pintura, Mattoni es nombrado vocal de la Comisión de Monumentos de la Provincia de Sevilla por fallecimiento de su maestro y antecesor en el cargo Eduardo

${ }_{13}$ González Ferrín, 2006: 641-663.

14 Debe tratarse de Manuel Portillo de Ávila y Herrera: Suárez Garmendia, 1986: 259. 
Cano. Ello le obligaba aún más con la conservación de la catedral como el primer monumento de la ciudad.

Por entonces, unos meses después de este último año, se crea la Junta Diocesana de Arte Religioso, al objeto de: "Velar por la conservación y restauración de las joyas artísticas e históricas de las iglesias del Arzobispado y proponer los medios adecuados al logro de dichos fines". La componían: Cayetano Fernández, chantre de la catedral y académico de Buenas Letras; Bartolomé Romero Gago, canónigo; Manuel de la Peña Fernández, presbítero, autor de la obra Arqueología Cristiana y catedrático de esta especialidad en el Seminario; Adolfo López, escultor y colaborador de Mattoni, y este mismo. Al año siguiente, con la ayuda del mencionado organismo, se restaura la valiosa sillería de coro del templo, que bajo la dirección de nuestro pintor y ejecución de los artistas Bellver, Solis y Adolfo López, se llevaría a cabo en los talleres establecidos en el Alcázar ${ }^{15}$.

Por otra parte, en 1906 se comenzó la rehabilitación de la capilla de la Virgen de la Antigua tras diecisiete años cerrada como consecuencia del incendio producido el 24 de marzo de 1889. La tarea corrió a cargo del ya anteriormente nombrado arquitecto Joaquín de la Concha Alcalde ${ }^{16}$. Con independencia de los trabajos técnicos llevados a cabo, debemos mencionar en esta ocasión el protagonismo de Mattoni cuando se planteó la posible restauración de los frescos aún existentes en los muros y las bóvedas de la capilla. Debieron ser los que menciona Carrillo y Aguilar representando un sol resplandeciente en cuyo interior se hallaba la Paloma del Espíritu Santo rodeada de ángeles, mientras algunos otros entonaban música y esparcían flores a la titular de la capilla. También pudieron ser los que alude el citado autor pintados al temple representando a San Pío Mártir y San Sabino entre decoración vegetal. Tal conjunto mural pudo ser pintado probablemente, según se estima, por Domingo Martínez entre 1734 y 1738 cuando decoró la capilla ${ }^{17}$. El arquitecto comprobó su estado y opinó: "no tan solo por su pésimo estado sino por el escaso valor artístico que a su juicio tenían, consideraba improcedente intentar su restauración". Solicitó, no obstante, el parecer de "personas peritas" en pintura de los componentes de la Junta Diocesana de Arte Religioso y también la del erudito inspector de las obras, Ricardo Velázquez Bosco. Mattoni, el más caracterizado de entre aquellos expertos y contando también con la opinión de su compañero Manuel de Torres, confirmó el aserto de Joaquín de la Concha en el sentido de desestimar su valor y por ende su restauración, dada - se dijo-su "mala ejecución y peor gusto artístico". Además -añadían- "Se hallaban muy deteriorados, hasta el punto de haber desaparecido por completo el dibujo en la mayoría de los trozos, creían casi imposible hallar artistas que se comprometieran a efectuar la restauración, por lo que opinaban que

\footnotetext{
15 Hernández González, 2014.

${ }^{16}$ Gómez de Terreros Guardiola, 1996: 223.

17 Valdivieso, 1990: 115.
} 
fueran picadas las pinturas existentes, dejando la piedra en su color natural"18. Unos meses después y en el trascurso de las tareas técnicas en la capilla, se comenzó la labor de restauración de los trozos de molduras de los cuadros que se destruyeron en el incendio y también las pinturas de los lienzos. Para inspeccionar las obras se creó una comisión compuesta por el propio Mattoni, Antonio de Alarcón y el arquitecto regionalista Mariano González Rojas. Posteriormente, en el mes de octubre, se procedió al picado y retundido de la bóveda y a la restauración de los lienzos, tarea que estuvo a cargo del pintor sevillano José de Escacena y Diéguez (1855-1909) bajo la "inmediata inspección" de Mattoni como secretario de la Junta. El Cabildo quedó muy satisfecho del trabajo realizado desinteresadamente y de la inspección llevada a cabo. Posteriormente, en las últimas décadas del siglo XX, las pinturas fueron objeto de una nueva restauración ${ }^{19}$.

Por entonces, el Cabildo eclesiástico designó una comisión, compuesta por el propio Mattoni, Gonzalo Bilbao, su hermano Joaquín, el erudito José Gestoso y el académico de Bellas Artes Cayetano Sánchez Pineda, para hacer una requisa de cuadros desconocidos y dar nueva colocación a los que tuvieran mérito de los existentes en la catedral. Efectuada la misma, se procedió a la instalación de la que dio cuenta Gestoso ${ }^{20}$. La labor documental de conocimiento, identificación y clasificación del patrimonio corrió a cargo sobre todo de este último con algunas sugerencias del académico citado, mientras la parte técnica de conservación y en su caso restauración o limpieza de cuadros estuvo en manos de Mattoni con la colaboración de los hermanos Bilbao Martínez.

Otra intervención en la catedral se produjo con posterioridad, en 1909, con motivo del citado movimiento sísmico que afectó a la iglesia del Sagrario, cuando se trató la ejecución de las obras para su reparación. No fue hasta mitad de junio de 1917 cuando la Junta de Obras se interesa de nuevo por el estado de conservación de este espacio anejo propiamente a la catedral. A este respecto, el arquitecto Joaquín de la Concha se compromete a la reparación de las cubiertas; mas, un año después, se supo que tal proyecto estaba pendiente de aprobación por el Ministerio correspondiente; no obstante, Mattoni propone que cuando se llevasen a cabo, se efectuase también el picado de los muros interiores para dejarlos expedito en su estado originario, a lo cual el citado director de obras se opone por su nula relación con el proyecto propuesto amén de su encarecimiento en equipo y jornales ${ }^{21}$.

Una de las últimas intervenciones de nuestro artista como miembro de la Junta de Obras de la catedral, se refiere, según la documentación, a la terminación de la "porción de fachada de estilo greco-romano comprendida entre las

\footnotetext{
${ }^{18}$ Gómez de Terreros Guardiola, 1996: 225 y 226.

19 Valdivieso, 1990: 109.

${ }^{20}$ Gestoso y Pérez, 1909: 10.

${ }^{21}$ Gómez de Terreros Guardiola, 1996: 223.
} 
puertas de San Miguel y de San Cristóbal"22. El arquitecto titular del templo, el ya citado Joaquín de la Concha, se hizo cargo en un principio de este nuevo proyecto, ya que a su parecer presentaba "un aspecto poco decoroso y digno que contrasta abiertamente con el aspecto suntuoso del resto del monumento", por lo que consideraba urgente su terminación ${ }^{23}$. Desde 1913 y durante un lustro se pretendía llevar a cabo la restauración de lo existente; sin embargo, en este ínterin, algunos integrantes de la citada Junta y al tiempo miembros de la Comisión de Monumentos, el también arquitecto Mariano González Rojas y el experto artista Virgilio Mattoni, censuraron lo que pretendía De la Concha: hermanar lo actual con el proyecto. Nuestro pintor manifestó que lo existente debería a todos los efectos suprimirse, ya que: "más moderna y de peor época que el resto de la Basílica debería desaparecer en vez de buscar su conservación y restauración, por ser un aditamento perjudicial al buen aspecto artístico por aquel lado de nuestra incomparable Catedral" ${ }^{24}$. Finalmente, en 1918, el autor del proyecto lo paralizó aun teniéndolo terminado, rehusando, antes de morir este mismo año, su envío al Ministerio para su curso. Su sucesor en el cargo, el arquitecto Francisco Javier de Luque, como director de las obras de conservación y restauración del monumento, retomó el asunto, elaborando su propio estudio tres años después con el consenso de los miembros veedores de las obras de la catedral, entre los cuales Mattoni era el más crítico aún sin ser arquitecto. Finalmente, las obras comenzaron en 1923, precisamente el año de su muerte, y terminarían un sexenio después para poder relucir con ocasión de la inauguración de la Exposición Iberoamericana.

Otro asunto de interés en el que intervino nuestro artista fue el de la reposición de vidrieras de la catedral. Se trataba de una cuestión importante que venía arrastrando desde 1888 , toda vez que no solo afectaba al cerramiento y protección del inmueble cara al exterior, sino también a la estética de las capillas o enclaves en que se encontraban situadas las vidrieras. Aunque desde 1907 preocupaba este asunto a los responsables de la integridad del templo, fue a comienzos del año siguiente cuando el arquitecto De la Concha expuso ante la Junta la situación en que se encontraban algunas de ellas y la inmediata reposición del conjunto. Se trataba de las correspondientes a las del frente sur y oeste del cimborrio y la más cercana a este del coro en el lado de la Epístola. En 1910 se propuso al mismo organismo la necesidad de ejecutar nuevas vidrieras para las capillas de la Virgen de la Antigua, San Hermenegildo y San José, además de la restauración de la situada en la capilla de San Antonio. Mattoni aprovechó la ocasión y manifestó su opinión técnica con motivo de las reposiciones que hacían falta de los ejemplares desaparecidos en 1888. Respecto a la primera de las citadas capillas, se colocó una

\footnotetext{
${ }^{22}$ Gómez de Terreros Guardiola, 1993: 245 y 261.

${ }^{23}$ Gómez de Terreros Guardiola, 1993: 247.

${ }^{24}$ Gómez de Terreros Guardiola, 1996: 229.
} 
nueva vidriera con la representación de San Fernando entronizado ejecutada por la casa Zettler de la Real Compañía de Vidrieras de Munich siguiendo un boceto de Mattoni ${ }^{25}$. Este, manifestó entonces que la correspondiente a la capilla de San Antonio había sido restaurada años atrás, sustituyéndose los vidrios coloreados de la parte superior por otros de tono blanco ${ }^{26}$.

\section{LA CREACIÓN ARTÍSTICA Y LITERARIA EN TORNO A LA CATEDRAL}

La profunda implicación de Mattoni en la conservación de la catedral le obliga con gusto a conocerla al dedillo tanto exterior como interiormente: sus fachadas, portadas, salas y dependencias con sus contenidos. Esta íntima relación, el valor histórico y artístico de los ejemplares incluidos, y la devoción popular a los mismos, que él compartía, le inspiran la creación artística y literaria de algunas de sus obras en las que se patentiza el binomio tradición-modernidad ${ }^{27}$.

En primer lugar y como un ejercicio de pintura nocturna, realizó en 1883 la obra titulada Una procesión de madrugada (Figura 1) ${ }^{28}$. Representa una atractiva panorámica del entorno de la catedral hispalense con el caserío colindante y gente en los balcones en el aura de una amanecida de Semana Santa en Sevilla. La crítica dijo que tenía estilo de Goya, "¡Lástima - añadía con cierto desdén- que estropee el cuadro la iglesia del lado derecho, ostentando tinta de cromo!"29. Interpreta los momentos fantásticos casi irreales del lento y silencioso desfile procesional con la Verónica en el centro durante la madrugada de un viernes santo sevillano al despuntar el nuevo día. La tenue luz del alba se conjuga con la trémula y expresiva de los cirios penitenciales para deformar las figuras y los objetos produciendo una imagen fantasmagórica cuando alguna túnica, capirote o bandera tremolando, cual fúnebres manchas negras a lo goyesco, se proyectan al unísono sobre el pavimento empedrado y los sillares del muro catedralicio.

Entre las obras del pintor existentes en diversas dependencias del templo metropolitano, debemos referimos en primer lugar por cronología a la que muestra el Proyecto de andas para la Virgen de los Reyes (1893), de la sacristía de la

${ }^{25}$ En algún lugar se cita a José Gestoso como el diseñador siguiendo el modelo del pendón. Guichot y Sierra, 1925: 110.

${ }^{26}$ Gómez de Terreros Guardiola, 1996: 216.

27 Tratamos exclusivamente las obras referidas o relacionadas con la catedral hispalense.

${ }^{28}$ Este cuadro, de formato apaisado y 79 x $140 \mathrm{~cm}$., fue presentado por su autor junto a otros cinco a la exposición que celebró la Academia Libre de Bellas Artes de Sevilla en 1883 en el local del ex convento del Ángel en la calle Rioja. Pérez Calero, 1998: 297. Se subastó en Ansorena, de Madrid, en mayo de 2012.

${ }^{29}$ La Andalucía, Sevilla, 27 de abril de 1883. 
Capilla Real de la catedral ${ }^{30}$. Tiene su antecedente en otro diseño de paso procesional para la patrona de Sevilla que el mismo Mattoni hizo dos años antes y que tampoco llegó a realizarse. El que nos ocupa, visto de perfil, venía a constituir el deseo del pintor de unir su propio fervor por la patrona de la ciudad con la regia devoción mariana de Fernando III y su primo San Luis teniendo como referencia a los reinos de Castilla, León y Francia. Todo ello, en esta ocasión, identificado en la persona de la donante $\mathrm{D}^{\mathrm{a}}$ María Luisa Fernanda, infanta de España y Duquesa de Montpensier por consorte. Para ello, respectivamente, inserta en las caídas del palio los escudos de los reinos peninsulares y la flor de lis como remate de los ocho varales. La obra tiene más valor documental que propiamente artístico.

En la Capilla de los Dolores se conserva un retrato de gran tamaño a cuerpo completo del Beato Diego José de Cádiz, ejecutado en 1894, año de su beatificación por el Papa León XIII. Profesó en el convento sevillano de su orden y Mattoni le tenía gran devoción por su pertenencia a los hermanos capuchinos, también le admiraba por su valiente oratoria en defensa de sus ideas conservadoras en materias de doctrina y moral. Le retrata idealmente, con su luenga barba y ojos entornados ante el altar, portando firmemente el crucifijo con ambas manos y la mitra en el suelo sobre un cojín. Se trata de una obra convencional de gran corrección dibujística, de tonalidad pastosa y aguda expresión del modelo.

Por su parte, la primorosa representación de La Anunciación (1897), de la Sacristía Mayor, es un óleo incorporado a un retablo neogótico profusamente decorado. La escena se desarrolla en una suntuosa estancia encortinada con dibujos de granadas y pavimento de variados y ricos motivos decorativos, que hacen recordar la escuela pictórica sienesa por la delicadeza mística y el lujo ornamental de la Madonna que mira al arcángel Gabriel arrodillado ante ella para mostrarle el Ave María. Mientras, al fondo, la escena se divide por una estilizada columna seudo salomónica con cimacio y capitel que deja ver en el alfeizar de la ventana una jarra de azucenas y a lo lejos una suntuosa iglesia de agudo chapitel neogótico en medio de una expresiva naturaleza matutina.

Esta tabla pintada es el resultado de la vinculación de Mattoni al arte ornamental fin de siglo, en el que se dan cita las artes y la industria decorativa que el propio pintor practicaba con la técnica del repujado. La interpreta con estética tardorromántica al modo espiritualista de John Ruskin, común por el pasado histórico y artístico medieval y protorrenacentista. Por ello, tiene implicaciones simbolistas afín al Arts \& Crafts (Artes y Oficios) de W. Morris y al gusto de prerrafaelistas como Rossetti o Edward Burne-Jones. Mattoni, pese a que nunca estuvo en la Europa continental ni en Inglaterra, aunque sí en Italia un cuarto de siglo antes, estaba al corriente de las modas artísticas de su tiempo a través de las ilustraciones en revistas que reproducían grabados de obras extranjeras y algunas

${ }^{30}$ Óleo sobre lienzo, de 80 x $70 \mathrm{~cm}$. Boceto firmado y fechado en 1893 y dedicado a S.A.R.I Serenísima Señora $D^{a}$ María Luisa Fernanda de Borbón. 
españolas $^{31}$. Precisamente, el catalán Alexandre de Riquer realizó años antes aunque con otra iconografía su composición titulada Anunciación (1893) ${ }^{32}$.

Por su parte, el lienzo que representa la Santísima Trinidad (1901), en la Capilla catedralicia de la Virgen de Belén, muestra iconográficamente al Padre acogiendo complacido al Hijo que se ofrece a Dios sosteniendo la cruz; ambos, sobre una nube blanquecina y vistiendo los colores litúrgicos. Es obra que, aun evocando la iconografía tradicional, manifiesta un simbolismo próximo al pietismo nazareno por la expresión algo fría y los gestos afectados de las figuras que la componen, a lo que contribuye la iluminación artificiosa que envuelve la escena ${ }^{33}$. Al propio tiempo, se emplea una factura suelta que propicia agilidad a la representación celestial.

Con motivo de las fiestas del Año Jubilar de 1904, se programó una serie de actos en la ciudad entre los cuales conviene ahora aludir a la realización de un cuadro colosal con la imagen de la Inmaculada Concepción, que sería colocado en la catedral. Para tal ocasión, el titular de la diócesis, Don Marcelo Spínola y Mestre, convocó a Mattoni y a sus colegas Genaro Parladé, Gonzalo Bilbao y José García Ramos, quienes de acuerdo con el arzobispo se comprometieron a llevar a cabo la ejecución de un cuadro de cinco metros de altura por tres de ancho, que sería pintado a la par en el estudio del Conde de Aguiar por él mismo y el propio Mattoni. Este, realizó una elaborada composición pictórica para tal ocasión en la que tuvo presente la iconografía de la cieguecita montañesina de la propia $\operatorname{seo}^{34}$ (Figura 2). A la postre, pasadas más de seis décadas, la obra sería ejecutada por el pintor sevillano Alfonso Grosso, esta vez con una iconografía alusiva a la Proclamación del Dogma de la Inmaculada y colocada sobre la puerta de la Concepción ${ }^{35}$.

31 Una de estas publicaciones, La Ilustración Artística, en su número 837 (10 de enero de 1898) se hace eco de cómo el prerrafaelismo y el misticismo artístico estaban en boga y habían tenido en Alexander de Riquer, desde los primeros días de su reaparición a uno de sus más fervientes partidarios, uno de sus más entusiastas apóstoles, uno de sus más activos propagandisticas.

Otras revistas ilustradas contemporáneas, que pudo conocer Mattoni, fueron: La Ilustración Ibérica y la inglesa The Studio, publicada desde 1894, cuando el mismo Riquer la trajo en su segundo viaje a Inglaterra.

${ }^{32}$ Oriundo de Calaf, pintó a la manera prerrafaelista de Burne-Jones en el presbiterio de la iglesia del monasterio de Montserrat.

También es probable que Mattoni tuviese conocimiento de la labor del Cercle Artistic de Sant Lluc, grupo católico neomedievalista fundado en Barcelona cuatro años antes, entre otros, por el propio Riquer.

${ }^{33} \mathrm{La}$ influencia de los nazarenos alemanes estuvo presente en la pintura sevillana desde mucho antes a través de la obra de pintores establecidos en Madrid como Antonio María Esquivel o Federico de Madrazo. Pérez Calero, 2012.

${ }^{34}$ Pérez Calero, 2006: 114.

${ }^{35}$ Este pintor ejecutó previamente varios bocetos para concluir su obra de ocho por seis metros alusiva a la proclamación del Dogma Inmaculadista. En el cuadro incluyó al 
Nuestro capacitado artista, ferviente católico y convencido monárquico, colaboró en 1908 en la ilustración de un primoroso álbum con láminas pintadas por los más acreditados artistas locales y que ofreció la Real Maestranza de Caballería a SSMM en la persona de la soberana $\mathrm{D}^{\mathrm{a}}$ Victoria Eugenia con motivo de una visita regia a la ciudad. La obra de Mattoni, titulada Sevilla monumental y religiosa, es de gran belleza. Representa los principales monumentos locales aleatoriamente agrupados entre los que figura en lugar preferente la catedral, de cuya Puerta de San Miguel sale la procesión del Gran Poder. Debemos ver en ella el deseo de su autor de mostrar una suerte de miscelánea artística de la ciudad tratada con purismo preciosista, como si de una amplia miniatura se tratase, en la que se dan cita arquitectura y escultura monumental animadas por el cortejo penitencial del primer plano (Figura 3).

A modo de referencia gráfica, tal vez con vista a ilustrar alguna publicación desconocida de carácter patrimonial, catálogo o inventario sobre la catedral, Mattoni representó dos piezas monumentales de gran valor que guarda el templo: la custodia de Juan de Arfe y el tenebrario. Se trata de pinturas convencionales tratadas con el habitual rigor arqueológico o artístico que tanto gustaba al autor (Figura 4).

Como ilustración del libro Quien no vio Sevilla..., publicado en 1920 por el Ayuntamiento de la ciudad, nuestro pintor dibujó la composición titulada $E l$ Corpus y la Inmaculada para acompañar al artículo que insertó el canónigo José Sebastián y Bandarán denominado La Ciudad de la Eucaristía y de la Inmaculada. Se trata de la exaltación de las dos solemnes fiestas vinculadas con la catedral. Bajo un arco apuntado gótico, Mattoni coloca de manera simbólica a dos seises de ambas advocaciones sosteniendo en altura un hermoso jarrón de azucenas mientras al fondo se ve la custodia de Juan de Arfe ${ }^{36}$.

Tenemos constancia de otras obras de propiedad particular con referencia a la catedral, algunas de las cuales son cuadros de interior que recuerdan versiones románticas de templos, capillas o monasterios a veces animados por fieles devotos. En esta línea se halla el cuadro titulado Interior de la Capilla de San Hermenegildo de la catedral de Sevilla, que posee sobre todo un valor documental al tiempo que precisión técnica al mostrar con rigor miniaturista y vivo colorido los pormenores del sepulcro del Cardenal Cervantes ${ }^{37}$.

Por su parte, el óleo sobre tabla que representa La salida de la procesión del Corpus por la Puerta de San Miguel de la catedral de Sevilla, es de estética tardorromántica por su pintoresquismo costumbrista ${ }^{38}$, pero puede también con-

\footnotetext{
citado arzobispo, defensor ardoroso de la doctrina concepcionista.

${ }^{36}$ Mattoni, 1920: 201.

${ }^{37}$ Óleo sobre lienzo, de 60 x $42 \mathrm{~cm}$. Propiedad de los herederos de D. Francisco Escalante, de Sevilla.

${ }^{38}$ Cuyo referente puede estar en la obra del mismo título de Manuel Cabral Bejarano de 1857 del Museo Nacional del Prado.
} 
siderarse propio del regionalismo artístico como una versión novecentista de la identidad cultural de la ciudad ${ }^{39}$.

Además de la pintura, Mattoni practicó con cierta asiduidad el ensayo y la poesía ${ }^{40}$. Nos interesa el primero a efecto de su relación con la catedral hispalense. Sabido es que escribió dos textos titulados Sevilla subterránea y El alma de la catedral de Sevilla. Para nuestro propósito nos detendremos en este último. Aunque en la actualidad perdido; sin embargo, en 1927, año en el que se unieron los nombres del Ateneo y la Generación literaria de ese año, la escritora Amantina Cobos, esposa del pintor Manuel Villalobos, conocedora de buena parte de su contenido, lo hizo público en una sesión necrológica en memoria del eximio y erudito Mattoni, compañero y amigo, fallecido cuatro años antes y vinculado a la citada institución. En esta ocasión, dio lectura a una parte del manuscrito inédito del artista. Mattoni traza con excelente verismo una visión sugerida ante la majestuosidad de la seo hispalense, donde veía surgir el alma del templo y a Felipe II llevado en andas por sus naves en procesión ceremonial conducida por todos aquellos magnates, prelados y artistas que, bien con sus ofrendas y celo o con su esfuerzo e inspiración, dieron principio y fin al magno edificio, del que el autor concretaba afirmaciones atinadas acerca de sus portentosas cualidades arquitectónicas y artísticas ${ }^{41}$.

Con el propósito de ilustrar semejante trabajo literario, que no vería la luz, Mattoni pintó el cuadro titulado El ángel y la catedral, protagonizado por un hermoso personaje alado que, portando una escudilla, se eleva por el exterior del monumento acompañado de varias palomas blancas en medio de una fulgurante luz solar sevillana ${ }^{42}$ (Figura 5).

La catedral y el Real Alcázar, ambos por su excepcional valor patrimonial y artístico, fueron los dos grandes monumentos de la ciudad que captaron especialmente el interés y la dedicación de Mattoni, pero fueron otros de menor entidad, la mayoría de ellos también medievales, los que igualmente le atrajeron como lecciones de historia del arte y fuente de inspiración para algunas de sus obras pintadas o escritas ${ }^{43}$.

Fecha de recepción: 13 de octubre de 2021.

Fecha de aceptación: 2 de mayo de 2021.

${ }^{39}$ Óleo sobre tabla, de 18 x $26 \mathrm{~cm}$. Propiedad de Antonio Galea, Sevilla.

${ }^{40}$ Información oral de Antonio Arroyo Mattoni, sobrino nieto del pintor. Lamentablemente no se conservan los escritos del artista o se encuentran actualmente en paradero desconocido.

${ }^{41}$ Anónimo, 1927.

42 De paradero desconocido.

${ }^{43}$ Entre ellos, la Casa de Pilatos, algunos monasterios como el de San Isidoro del Campo en Santiponce y el de Santa Paula, el Palacio de San Telmo, el Hospital de Venerables Sacerdotes, el Museo de Bellas Artes, así como numerosas iglesias parroquiales de la ciudad. 


\section{BIBLIOGRAFÍA}

AAVV. (1920) José Gestoso (1852-1917) y Sevilla: Erudición y patrimonio. Sevilla: Editorial Universidad de Sevilla.

Anónimo (1927): "Ateneo. Sesión Necrológica en memoria de Virgilio Mattoni”. En: El Noticiero Sevillano, Sevilla, 3 de febrero.

Gestoso y Pérez, José (1909): "Una requisa de cuadros en la catedral de Sevilla". En: El Correo de Andalucía, 6, 7 y 8 de enero.

Gómez de Terreros Guardiola, María del Valle (1993): “Obras de restauración y terminación de la fachada del ángulo suroeste de la catedral de Sevilla. El proyecto de Francisco Javier de Luque.” En: Laboratorio de Arte, 6, pp. 245-262. (1996): "Obras de Joaquín de la Concha Alcalde en la catedral de Sevilla". En: Laboratorio de Arte, 9, pp. 209-237.

González Ferrín, María Isabel (2006): "Documentación de la Junta de Obras en el Archivo Catedral de Sevilla”. En: Álvarez Márquez, M. Carmen/Romero Tallafigo, Manuel/Rubio Merino, Pedro (coords.). Archivos de la Iglesia de Sevilla, homenaje al archivero D. Pedro Rubio Merino. Córdoba: Caja Sur, pp. 641-664.

Guichot y Sierra, Alejandro (1925): El Cicerone de Sevilla. Monumentos y Artes Bellas. Sevilla: Imprenta de Álvarez, tomo I.

Hernández González, Salvador (2014): La escultura en madera del gótico final en Sevilla. La sillería de coro de la Catedral de Sevilla. Sevilla: Diputación de Sevilla.

Mattoni de la Fuente, Virgilio (1890): Discurso leído con motivo de la concesión de premios del curso 1889-1890 a los alumnos de la Academia y Escuela de Bellas Artes de Sevilla. Sevilla.

(1920): Quien no vio a Sevilla... Sevilla: Excmo. Ayuntamiento de Sevilla.

Pérez Calero, Gerardo (1976): "Virgilio Mattoni y las corporaciones artísticas sevillanas”. En: Boletín de Bellas Artes. Real Academia de Bellas Artes de Santa Isabel de Hungría de Sevilla, IV, pp. 181-198.

(1977 y 1996): El pintor Virgilio Mattoni. Sevilla. Colección Arte Hispalense, $\mathrm{n}^{\circ}$ 15. Sevilla: Diputación de Sevilla.

(1998): "La Academia Libre de Bellas Artes de Sevilla (1872-1888)". En: Laboratorio de Arte, 11, pp. 275-300.

(2000): "Obras inéditas simbolistas y nazarenas del pintor V. Mattoni”. En: Laboratorio de Arte, 13, pp. 409-418.

(2012a): "Virgilio Mattoni de la Fuente (1842-1923)". En: Diccionario Biográfico Español. Madrid: Real Academia de la Historia, vol. XXXIV.

(2012): "Consideraciones estéticas en torno a la obra del pintor Antonio M. Esquivel". En: Laboratorio de Arte, 24, pp. 527-535.

(2015): Mattoni de la Fuente, Virgilio. En: Universidad de Sevilla. Personalidades. Sevilla: Editorial Universidad de Sevilla. 
(2020): "Virgilio Mattoni y el Real Alcázar de Sevilla: entre la erudición y la pintura". En: Laboratorio de Arte, 32, pp. 413-432.

Suárez Garmendia, José Manuel (1986): Arquitectura y urbanismo en la Sevilla del siglo XIX. Sevilla: Diputación de Sevilla.

Valdivieso González, Enrique (1978): Catálogo de las pinturas de la catedral de Sevilla. Sevilla: Enrique Valdivieso.

Valdivieso González, Enrique (1990): "Pinturas de Domingo Martínez en la capilla de la Virgen de la Antigua en la catedral de Sevilla". En: Laboratorio de Arte, 3, pp. 109-122. 


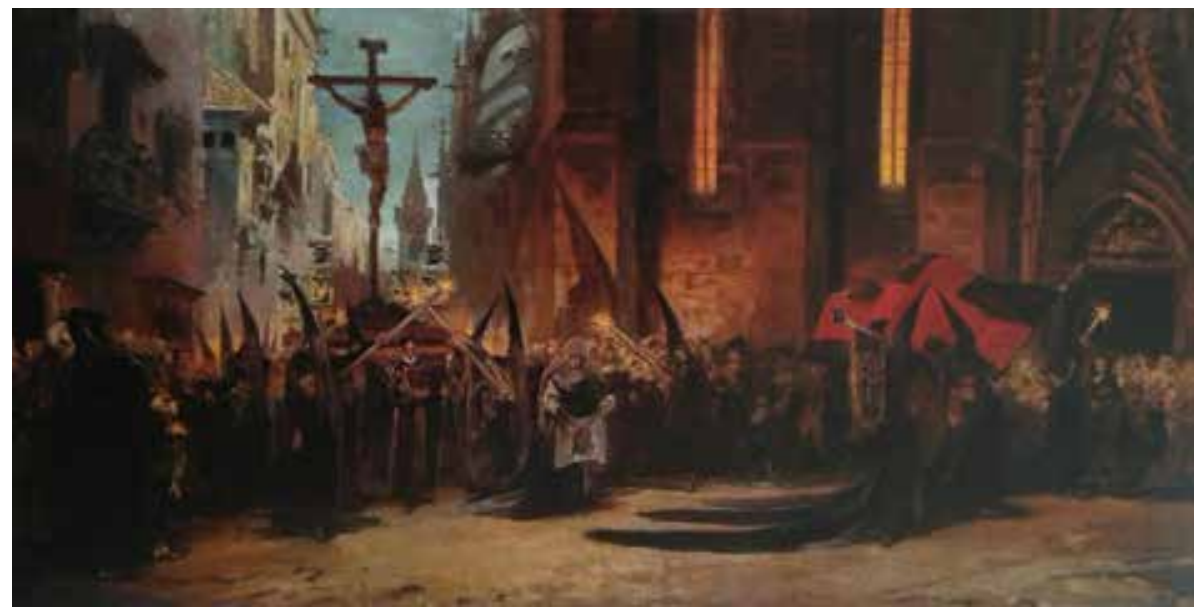

Figura 1. V. Mattoni. Una procesión de madrugada. 1883. Colección particular. 


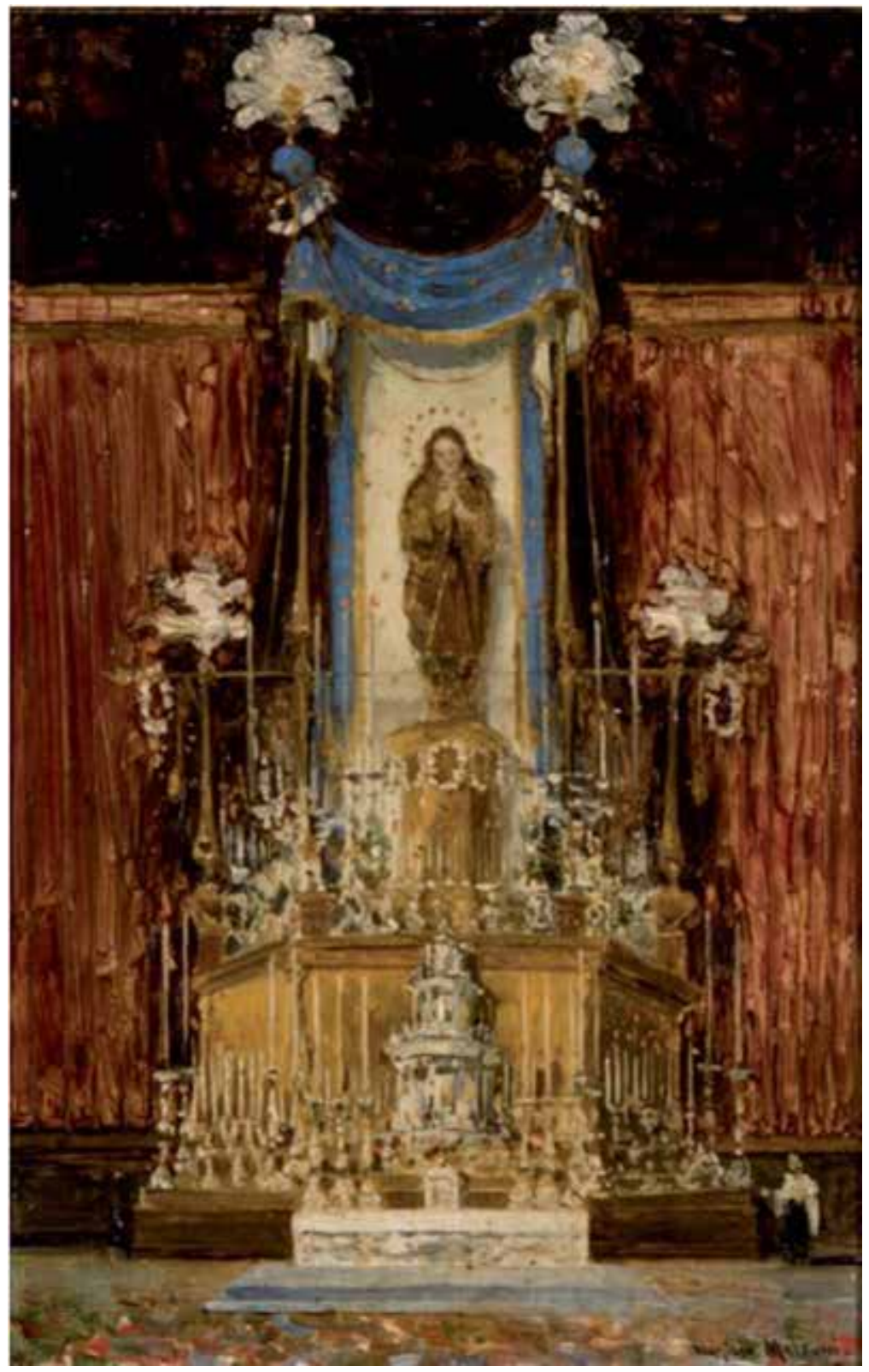

Figura 2. V. Mattoni. Altar de la Inmaculada. Colección particular. 


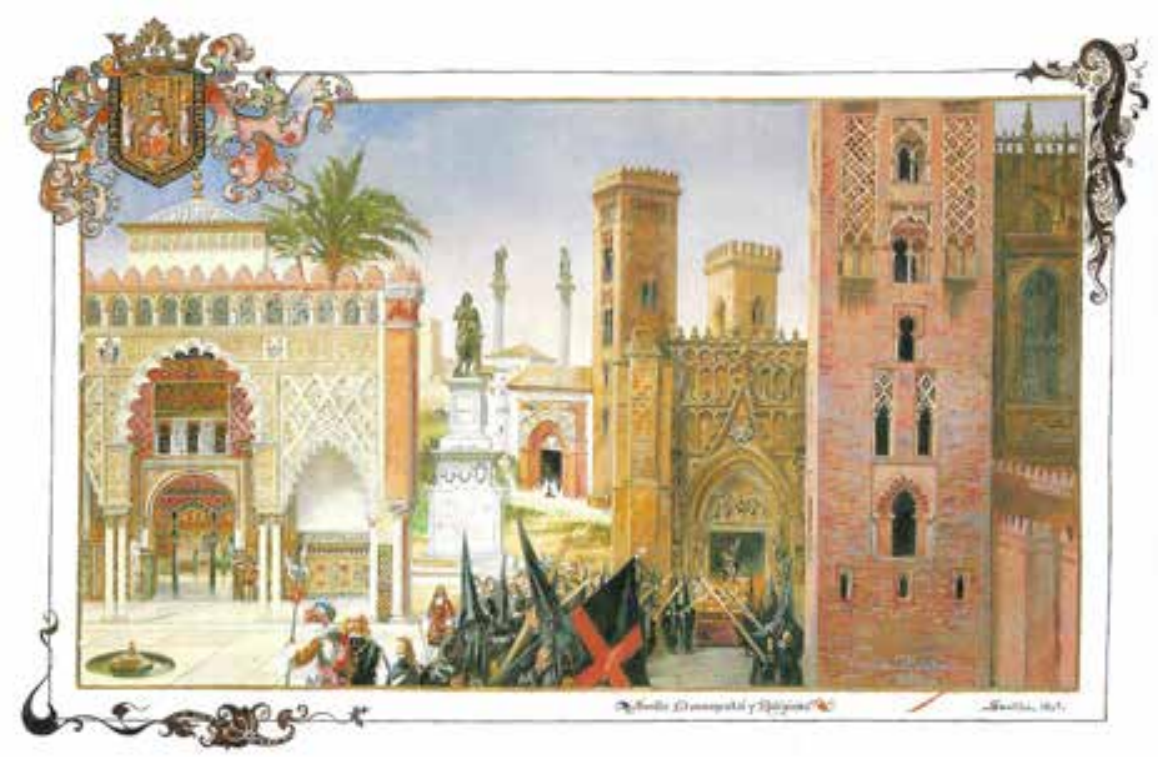

Figura 3. V. Mattoni. Sevilla monumental y religiosa. 1908. Lámina del album ofrecido a S.M. la reina Victoria Eugenia por la Real Maestranza de Caballería de Sevilla. 

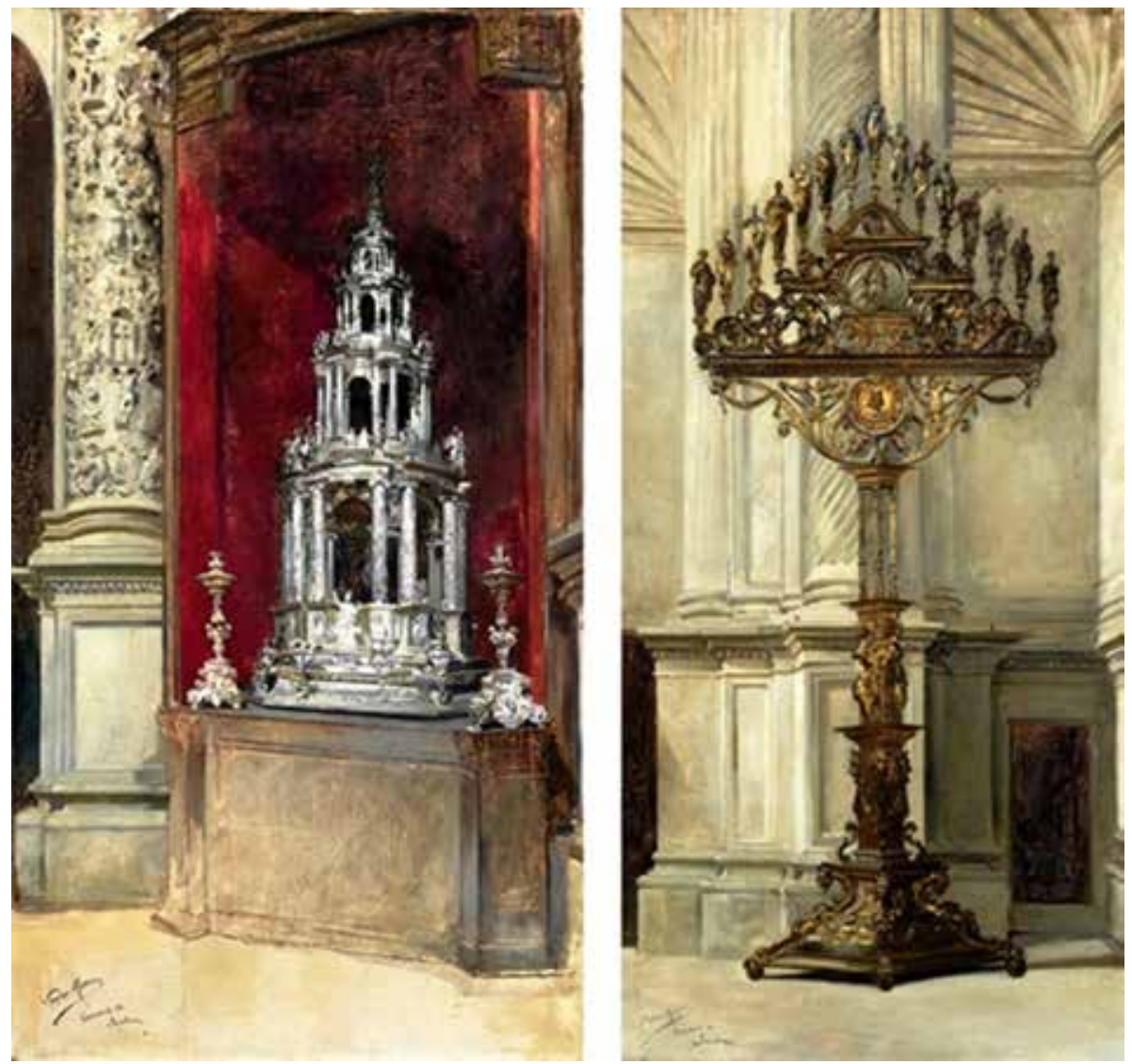

Figura 4. V. Mattoni. La custodia y el tenebrario de la catedral de Sevilla. Colección particular. 


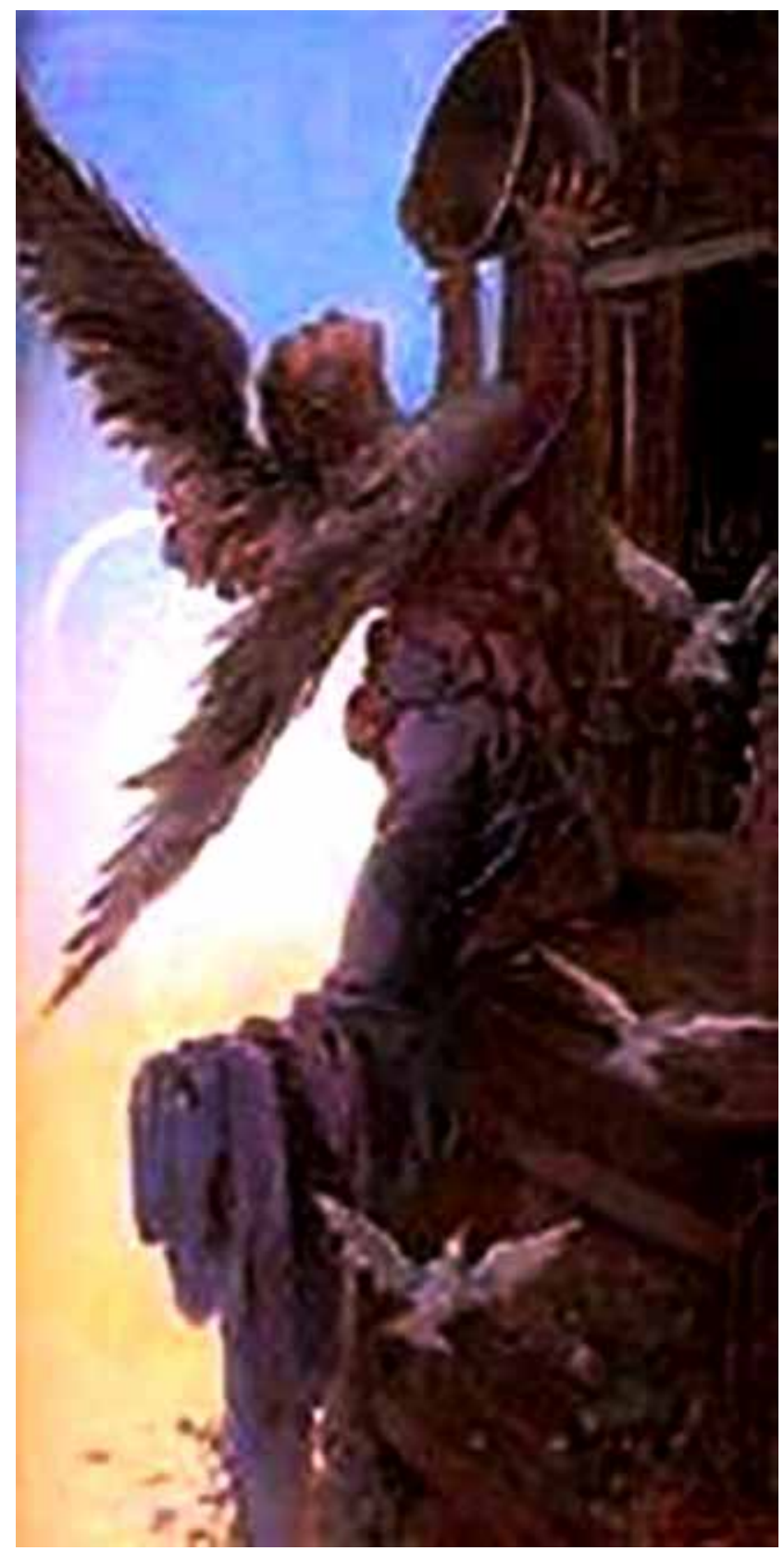

Figura 5. V. Mattoni. Ilustración para su obra inédita El alma de la catedral. Colección particular. 